\title{
Neuroethological Approach to Understanding Intelligence
}

\author{
DaeEun Kim \\ Biological Cybernetics Lab \\ School of Electrical and Electronic Engineering \\ Yonsei University \\ Seoul, 120-749, South Korea
}

\begin{abstract}
The neuroethology is an interdisciplinary study among artificial intelligence, biology and robotics to understand the animal behavior and its underlying neural mechanism. We argue that the neuroethological approach helps understand the general artificial intelligence.
\end{abstract}

\section{Introduction}

Many animal behaviours demonstrate unsolved mysteries and some biologists have studied to answer the question of how their intelligence is connected to the behaviours. Their intelligence level is not superior to human intelligence, but the study of animal intelligence provides a clue to understand what kind of neural mechanism supports the intelligence. This study stimulates relevant studies in the field of robotics and artificial intelligence. Robotists have worked to find how sensory stimuli are connected to motor actions, what is a good mapping from sensors to motors, how the learning mechanism helps to build more complete information about the environment, and what is the optimal control based on the acquired environmental information. All these kinds of problems can also be found in the analysis of animal behaviours as well as human brain system. Still unknown are their underlying mechanisms in details.

Some scientists simulate human brain models to understand the general intelligence, and analyze a map of neurons in terms of the function of human brain, and try to answer how the system of neurons works. Assuming all biological animals have diverged from their ancestral animals with respect to the evolutionary track, we can guess that their brain system and the functions should have similar neural mechanisms and operations. The neuroethological approach explains how and why the animals behave at a specific situation or survive their environments. It shows how their brain system organizes the sensory-motor loop and issues appropriate commands of control for desirable behaviours, and ultimately may explain what is the intelligence. From invertebrate animals to humans, the intelligence follows evolutionary steps as the complexity of the brain system develops. We believe that this kind of study can demonstrate all the levels of intelligence, and furthermore, elements of artificial general intelligence.

\section{Neuroethological Approach}

The neuroethology, as a multidisciplinary study between neurobiology and ethology, focuses on the study of interaction of neural circuitry to explain the behavioural concepts or strategies found in animals. This field covers the underlying mechanism and analysis to cover high-level features of animal cognition. There is an example of neuroethological approach to understand a part of the artificial general intelligence. Passive sensing and active sensing depending on motor actions are classified as a different level of intelligence. Passive sensing implies receiving sensory information from the environment without any sensor-motor coordination. In contrast, active sensing is involved with a sequence of motor actions which change the environment or activate the body movement, and ultimately produces another view of sensory information.

A collection of sensory information depending on a sequence of motor actions provides information of the environment in more details. For example, bats have an echolocation system to search for food. They use the ultrasonic system to emit the signal and sense the reflected sonar signal to measure the location, size and the moving speed of their prey. Similarly, electric fish have electrolocation by generating the electric field and observe the change of the electric field distorted by the surrounding objects. These animals have a relatively large size of the brain to process the active sensing results. It is believed that their brain systems have a prediction model for their self-generated motor actions to estimate accurately the location of their target object. This system corresponds to a forward model in the human brain system. The cerebellum includes the forward model and inverse model for their motor actions and sensory information (WK98). Also, it is presumed that crickets show a low-level forward model for auditory system (Web04). From the neuroethological analysis, we can infer the fundamental mechanism of the brain system and thus a predictive system for intelligence can be explained.

Biologists and neuroethologists have studied sensory 
systems and their sensorimotor coordination (Car00). Bats use echolocation for prey capture and navigation in the cave, and electric fish show underwater navigation and electric communication. Barn owls have their specialized auditory system. Aplysia show the fundamental mechanism of learning and memory with a small number of neurons. Rats use spatial cognition and memory, and navigate in the dark. Crabs show visual information processing, the eye-leg coordination and path integration (ZH06). Honeybees have visual navigation, learning and its flight mechanism depending on vision (SZ00). Many animals have their own features of sensory mechanism, the coordination of motor actions, or adaptibility and learning mechanism, and many of them can partly provide a key to understand the intelligence.

Animals have robust mechanism adapting themselves to their environment. Their sensory processing and pattern recognition are organized to extract relevant information from the environmental system. The system robustly works even in noisy environments. The motor actions triggered by sensory information are regulated efficiently and a sequence of multiple motor actions are integrated in high skills. This kind of dexterity should be explained by neural mechanism to understand the intelligence.

\section{Robotics}

There have been many robotic applications that look intelligent to the public. However, in many cases, the approach is far from the artificial general intelligence. Robotic problems consider a specific application and they are often based on engineering analysis and design. Intelligence is a side-effect with the approach, but neurorobotics and neuroethological approach to robotics have demonstrated the neural mechanism and its application motivated by biological systems. It is a part of systematic and integrative approach to the intelligence. The current state of the work still has a narrow range of scope for the artificial general intelligence. If more integrative methods are available to explain high-level features of intelligence such as planning and inference, it would help understand the general artificial intelligence.

Intelligent robots handle how a robotic agent behaves autonomously. The robotic agent behaviours resemble the animal behaviours with respect to the system model including sensory system, motor actions, sensory-motor coordination, and further, a hierarchy of the interaction levels among the system elements. Thus, understanding animal behaviours may lead to a possible application of intelligent robotic system. In addition, understanding human brain system can produce intelligent machines with human-level cognition.

\section{Artificial General Intelligence}

The general intelligence needs to cover all levels of intelligence from low to high level of features. How the system is organized into a high level of features is still an open question. Another program or concept is required for the artificial general intelligence. How the agent generalizes the knowledge over various domains or modalities and how the agent conceptualize the instances are interesting problems in this field. The process should be modelled with a neuronal system and it is believed that it requires a complex type of adaptation or networked organization. We expect the features could be explained by complex networks over a collection of neuron units, where each neuron has its own adaptivity and learning. A simple neuron itself has adaptation for the synaptic plasticity depending on their inputs and output. This kind of adaptation may lead to a complex structure of agents and explain how agents interact each other. It seems it may be related to emergent properties of a collection of units.

This neuroethological study may reveal the basic element of the artificial general intelligence for learning and adaptation process. Especially it has a potential of application for robotic agents to behave intelligently as natural animals do. Yet we still need further work to relate the neuroethology to the high-level agent architecture including reasoning, planning and solving problems.

\section{Conclusion}

We suggest a neuroethological approach to robotics can explain the behaviour-level intelligence and its underlying neural mechanism. It will explain many important concepts of sensory integration, sensor-motor coordination and learning mechanism needed for the general artificial intelligence.

\section{Acknowledgments}

This work was supported by the Korea Science and Engineering Foundation(KOSEF) grant funded by the Korea government(MEST) (No. 2009-0080661)

\section{References}

T.J. Carew. Behavioral Neurobiology. Sinauer Associates, 2000.

M.V. Srinavasan and S. Zhang. Honeybee navihation: Nature and calibration of the odometer. Science, 287:851-853, 2000.

B. Webb. Neural mechanisms for prediction: do insects have forward models? Trends in Neurosciences, 27:278-282, 2004.

D. W. Wolpert and M. Kawato. Multiple paired forward and inverse models for motor control. Neural Networks, 11:1317-1329, 1998.

J. Zeil and J.M. Hemmi. The visual ecology of fiddler crabs. J. of Comparative Physiology A, 192:1-25, 2006. 